\title{
A highly stable noncontact SPM for surface profile measurement and its application to insulating samples
}

\author{
Shigeaki GOTO*, Minglei LI*, So ITO*, Yuki SHIMIZU* and Wei GAO* \\ *Department of Nanomechanics, Tohoku University \\ Aramaki Aza Aoba 6-6-01, Sendai, Miyagi, 980-8579, Japan \\ E-mail: goto@nano.mech.tohoku.ac.jp
}

Received 28 February 2016

\begin{abstract}
A noncontact scanning electrostatic force microscope (SEFM) for surface profile measurement is introduced, and applied to the profile measurement of insulating samples. The SEFM calculates the tip-sample distance with an algorithm named 'dual-height method', and compensates for the fluctuation of the tip-sample distance to obtain accurate surface profile image. Glass and acrylic, which are commonly applied to optical components, are prepared as the insulating samples, and bias voltage is applied to the samples with an electrode clamp. On the glass sample, electrostatic force is observed in the case that the bias voltage of $50 \mathrm{~V}$ is on. On the acrylic sample, however, electrostatic force is not observed regardless of the bias voltage. Surface profile measurement of the glass sample is demonstrated. The tip-sample distance image is created with the dual-height method from the data of the tip trajectories and the frequency shift signals obtained through the constant tip-sample interaction scan. The results show that the tip-sample distance is kept larger than $100 \mathrm{~nm}$, which is more than ten times larger than that of conventional noncontact SPMs. The large tip-sample distance brings the SEFM a high robustness against the disturbances from environment, which would cause tip-sample collision in the conventional noncontact SPMs. The same area on the same sample is measured also in an atomic force microscope (AFM) mode, which is realized by setting the bias voltage $0 \mathrm{~V}$. The SEFM image agrees with the AFM image quantitatively, showing that the SEFM with the dual-height method is applicable to noncontact profile measurement of the glass. On the other hand, it is difficult to utilize the SEFM in the profile measurement of the acrylic sample because the electrostatic force on the acrylic is not controllable with the voltage of the electrode clamp.
\end{abstract}

Key words : Scanning probe microscope, Electrostatic force, Noncontact, Profile measurement, Glass, Acrylic, Tuning fork, Optical components, Atomic force microscope

\section{Introduction}

Recently, artificial microstructured surfaces, which have been realized by ultra-precision machining techniques, are applied to many kinds of optical components: light guide panels in liquid crystal displays, gratings in precision position sensors, and so on. The functions and the performances of the microstructures are designed by the cross-sectional views of the surface profiles as well as by the top views of the surface patterns. In order to evaluate the machining accuracy, three-dimensional surface profilers for the microstructure have therefore been demanded.

Scanning probe microscopes (SPM), which were first invented by Binnig et al. (1982, 1986), have been attracting interests as the surface profilers for the microstructures. In contrast to other microscopes such as optical microscopes and scanning electron microscopes (SEM), the SPMs have an advantage of an ability to obtain three-dimensional surface profile images in nanometric resolution without necessity of cutting the sample. Especially, the noncontact SPMs such as scanning tunneling microscope (STM) and noncontact atomic force microscope (AFM) have high potential for nondestructive measurement because they can, in principle, scan the surface without scratching/wearing damages to the sample and the tip. The noncontact SPMs utilize tip-sample interactions such as atomic force and tunneling current, 
whose intensities vary as functions of the tip-sample distance. In many examples of SPMs for surface profile measurements, as reviewed by Giessibl (2003), the SPMs are operated in the "constant interaction mode" in which the tip-sample interactions are maintained constant by feedback-controlling the tip-sample distance. In this mode, the tip trajectories are recorded as the surface profiles. The tip trajectories show good agreement with the surface profiles because tunneling current and atomic force are so weak that the tip-sample distance is maintained to be less than $5 \mathrm{~nm}$ (Barth et al., 2011). The small tip-sample distance, however, requires nanometric positioning stability of the tip to avoid the tip-sample collision. The conventional noncontact SPMs are therefore sensitive to mechanical or electromagnetic disturbances from the experimental environment. Also the enhancement of the scanning speed increases the feedbackpositioning error of the tip, resulting in the damages of the tip and the sample.

As another choice for the tip-sample interaction for noncontact SPMs, we have been studying electrostatic force. The electrostatic force, also called Coulomb force, is a tip-sample interaction induced by the voltage potential difference between the tip and the sample. The electrostatic force is a powerful and long-range interaction compared to the atomic force and the tunneling current. The long interaction range enables the SPM to scan the surface with larger tip-sample distance than $100 \mathrm{~nm}$. Hu et al. (1995) reported about the investigation of the electrostatic tip-sample interaction expecting that the electrostatic force brings the SPMs high robustness to the disturbances by reducing the risk of the tipsample collision. However, it is difficult to secure the accuracy of the profile image obtained by the conventional scanning electrostatic force microscopy (SEFM) because the tip-sample distance, which must be constant in the constant interaction mode, would fluctuate influenced by the surface distribution of the electrostatic force. The conventional SEFMs have therefore been utilized in the investigation of electrostatic property of surfaces with already-known profiles.

In order to satisfy both the stability and the accuracy, the authors proposed a novel principle of SEFM for surface profile measurement (Gao et al., 2012). The proposed SEFM calculates the tip-sample distance with an algorithm named 'dual-height method' to compensate for the fluctuation of the tip-sample distance. The experiment of surface profile measurement of a metal sample (gold-coated glass sample) was demonstrated. During the measurement, the tip-sample distance was maintained larger than $100 \mathrm{~nm}$, realizing a highly stable noncontact scan. And the profile image obtained by the SEFM agreed with that obtained by a commercially available AFM quantitatively. In the initial study, we limited the application of the SEFM to non-insulating (conductive or semiconductive) materials because the system to apply the bias voltage is similar to that of the STM, which can't obtain surface profile of insulating samples. In a subsequent report of our study (He et al., 2014), however it was confirmed that the electrostatic force was observed also on a glass sample which was clamped by an electrode. This indicated a possibility that the SEFM is applicable to insulating materials as well as metal.

This paper reports the experimental investigation of the feasibility of the SEFM in noncontact surface profile measurement of insulating materials. Glass and acrylic, which are commonly applied to the optical components, are prepared as the sample. Surface profile measurement is carried out to examine whether the compensation algorithm of the dual-height method works to obtain quantitatively accurate surface profiles of insulating samples.

\section{Principle}

\subsection{Electrostatic force}

Conventional SPMs obtain the surface profiles by recording the trajectory of the tip scanning over the sample surface. And the tip-sample distance has to be maintained constant during the scan to conform the tip trajectory to the surface profile. The tip-sample distance of SEFM would, however, fluctuate depending on the intensity distribution of the electrostatic force on the sample surface. The accuracy of the profiles obtained from the tip trajectory of the SEFM is therefore worse than that obtained with the conventional SPMs.

The proposed SEFM compensates for the tip-sample distance fluctuation to obtain accurate surface profiles. The algorithm named "dual-height method" calculates the tip-sample distance based on the nature of electrostatic interaction. This section firstly introduces the nature of the electrostatic force, and then explains the principle of the dual-height method.

Figure 1 shows a schematic of the electrostatic interaction. Assuming that voltage difference between the tip and the sample are kept constant by connecting the tip and the sample to a constant voltage source, the electrostatic force $F$ is expressed as the $\mathrm{Z}$ directional derivative of the energy $W$ stored in the tip-sample capacitance $C$ as: 


$$
F=W^{\prime}=\frac{1}{2} C^{\prime}\left(V_{d c}+V_{c p d}\right)^{2}
$$

where $W^{\prime}=\partial W / \partial z, C^{\prime}=\partial C / \partial z$ and $V_{d c}$ is the voltage difference between the tip and the sample. $V_{c p d}$ is the contact potential difference (CPD), which contributes to the intensity distribution of the electrostatic force in XY plane. For a sharp tip with a spherical apex of radius $R, F$ is known to be expressed as:

$$
F=\pi \varepsilon_{0} \varepsilon_{r}\left(V_{d c}+V_{c p d}\right)^{2} \frac{R}{h}
$$

where $\varepsilon_{0}$ is the dielectric constant of vacuum, $\varepsilon_{r}$ is the relative static permittivity and $h$ is the tip-sample distance (Belaidi et al., 1997). Electrostatic force is commonly sensed by frequency modulation (FM) techniques considering the resolution and the response. An SEFM probe unit is composed of a resonator and a tip. The effective resonance frequency $f_{\text {eff }}$ of the probe unit is expressed as:

$$
f_{\text {eff }}=\frac{1}{2 \pi} \sqrt{\frac{k-F^{\prime}}{m}}
$$

where $k$ is the spring constant of the resonator, $m$ is the equivalent mass of the oscillation system and $F^{\prime}$ is $\mathrm{Z}$ directional derivative of the external force acting on the tip. Using first-order Taylor approximation, the frequency shift $\Delta f$ of the resonance from the fundamental resonance frequency $f_{0}$ is expressed as:

$$
\Delta f=f_{\text {eff }}-f_{0} \approx \frac{f_{0}}{2 k} F^{\prime}
$$

where

$$
f_{0}=\frac{1}{2 \pi} \sqrt{\frac{k}{\mathrm{~m}}} .
$$

From Eqs. (2), (4) and (5), the frequency shift induced by electrostatic force is expressed as

$$
\Delta f \approx-\frac{\pi \varepsilon_{0} \varepsilon_{r} R f_{0}}{2 k}\left(V_{d c}+V_{c p d}\right)^{2} \frac{1}{h^{2}} .
$$

Accordingly, the electrostatic force between the tip and the sample is inversely proportional to the tip-sample distance, and the frequency shift of the probe induced by the electrostatic force is inversely proportional to the square of the tipsample distance.

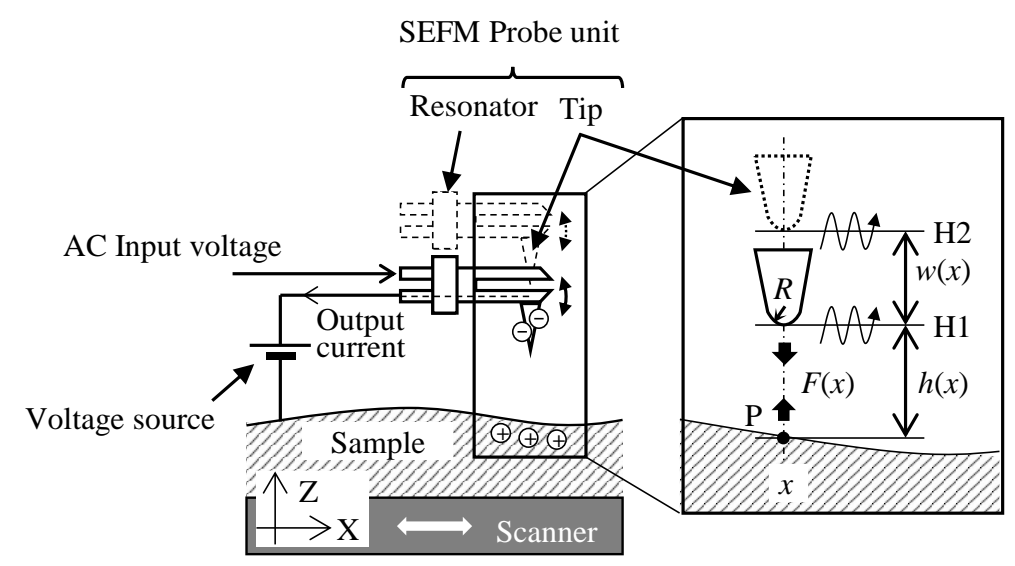

Fig. 1 A schematic of the tip-sample electrostatic interaction.

\subsection{Dual-height method}

The dual-height method, which was proposed for the SEFM profile measurement, is an algorithm to calculate the tip-sample distance. As illustrated in Fig. 1, the dual-height method requires a pair of electrostatic force signals sampled at different two tip-sample distances. Assuming that the frequency shift signal obtained at the height H1 is denoted by Eq. 6, the frequency shift $\Delta f_{w}$ at the other height $\mathrm{H} 2$ is expressed as:

$$
\begin{aligned}
& \Delta f_{w}=-\frac{\pi \varepsilon_{0} \varepsilon_{r} R f_{0}}{2 k} V_{d c}^{2} \frac{1}{(h+w)^{2}} \\
& w=d_{b}-d_{a}
\end{aligned}
$$


where $d_{a}$ and $d_{b}$ are $\mathrm{Z}$ directional position of the tip which are recorded as the displacement of the $\mathrm{Z}$ scanner at the heights $\mathrm{H} 1$ and $\mathrm{H} 2$, respectively. From Eq. (6) and Eq. (7), the tip-sample distance is calculated as:

$$
h=w \frac{\sqrt{\Delta f_{w} / \Delta f}}{1-\sqrt{\Delta f_{w} / \Delta f}} .
$$

It should be noted that all the parameters including the voltage potential are cancelled in Eq. 9. The absolute value of the tip-sample distance thus can be calculated with accurately cancelling the intensity distribution of the electrostatic force.

Figure 2 illustrates the way to apply the dual-height method to the surface profile measurements. The SEFM scans the surface in constant tip-sample interaction mode, in which the $\mathrm{Z}$ directional position of the tip is feedback controlled to keep the frequency shift constant. The scans are conducted twice per a scanline, and the setpoints of the feedback is switched between two values at the all turning points. In this way, a pair of frequency shift signals at different two distances can be sampled at any position in X. The tip-sample distance at any position in X can thus be calculated from Eq. (9). Then, the surface profile $s(x)$ is calculated as:

$$
s(x)=d_{a}(x)-h(x) .
$$

The dual-height method compensate for the fluctuation of the tip-sample distance regardless of the factor of the fluctuation. Even though the feedback error in $\mathrm{Z}$ direction, as well as the force intensity distribution in XY plane, induces the fluctuation of the tip-sample distance, the SEFM obtains accurate surface profile without the influence of the force intensity distribution nor the feedback error. In the evaluation of 3D topographies, the scan is repeated in Y direction to create three-dimensional image.

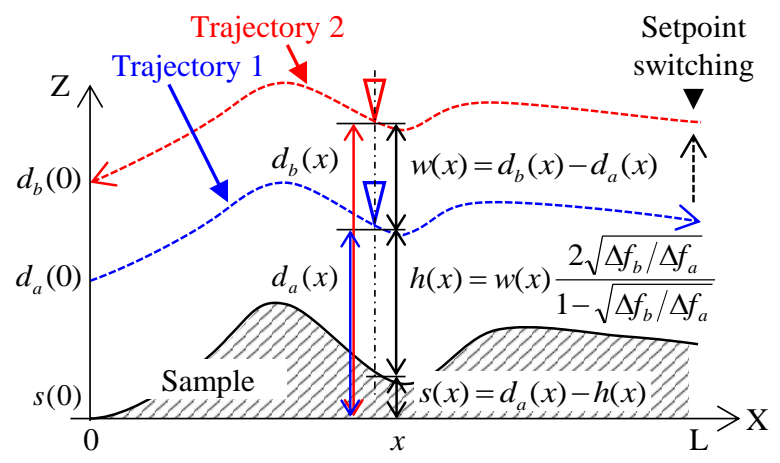

Fig. 2 A schematic diagram to illustrate the sequence of a single profile measurement with the dual-height method. The SEFM scans the sample surface in the constant interaction mode. The surface profile is calculated from the data of tip trajectories and the frequency shifts recorded during the scan.

\section{Experiment setup}

Figure 3 shows a schematic of the developed SEFM. The SEFM consists of an oscillation controller (OC4, Nanonis), a Z scanner, a probe unit, an XY scanner (P-621.2CL, Physik Instrumente), a current-to-voltage converter and a constant voltage source with an electrode clamp. The oscillation controller includes two functional components: a 'self-oscillating circuit' to oscillate the probe unit at the actual resonant frequency, and a 'phase-locked loop circuit' to demodulate the oscillating frequency into DC signal. The combination of these two components converts the frequency shift of the resonance of the resonator into DC signal, which corresponds to the Z-directional gradient of the force acting on the tip.

Figure 4 shows a photograph of the Z-scanner and SEM images of the probe unit. The Z-scanner consists of a linear encoder and a long-stroke PZT actuator, realizing a measurement resolution of $0.5 \mathrm{~nm}$ with an accuracy of $5 \mathrm{~nm}$ throughout the stroke of $50 \mu \mathrm{m}$ in $\mathrm{Z}$ (Goto et al., 2011). The probe unit consists of a tuning fork quartz crystal resonator (TFQCR) and two electropolished tungsten tips. One of the tips is for sensing the force, and the other is a counter weight for balancing the mass to secure a high Q factor ( $\mathrm{Ng}$ et al., 2009). The resonant frequency and Q-factor of the probe unit were approximately $29 \mathrm{kHz}$ and 6,000, respectively. The tips are electrically connected to the surface electrode of the TFQCR with conductive epoxy in order to set the voltage potential of the tip to approximately $0 \mathrm{~V}$ through the virtual short of the Op-Amp used in the current-to-voltage converter. 


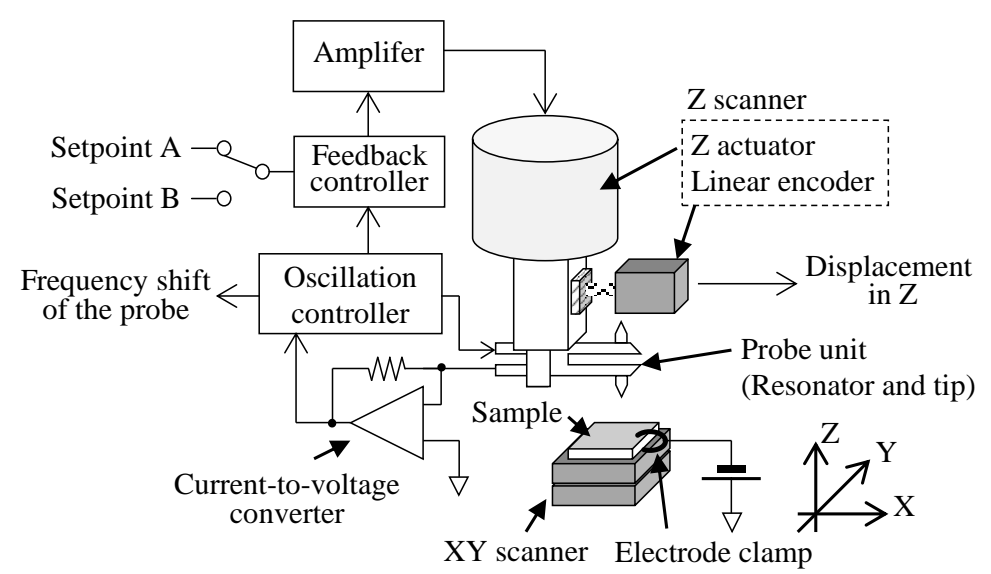

Fig. 3 A schematic of the developed scanning electrostatic force microscope.

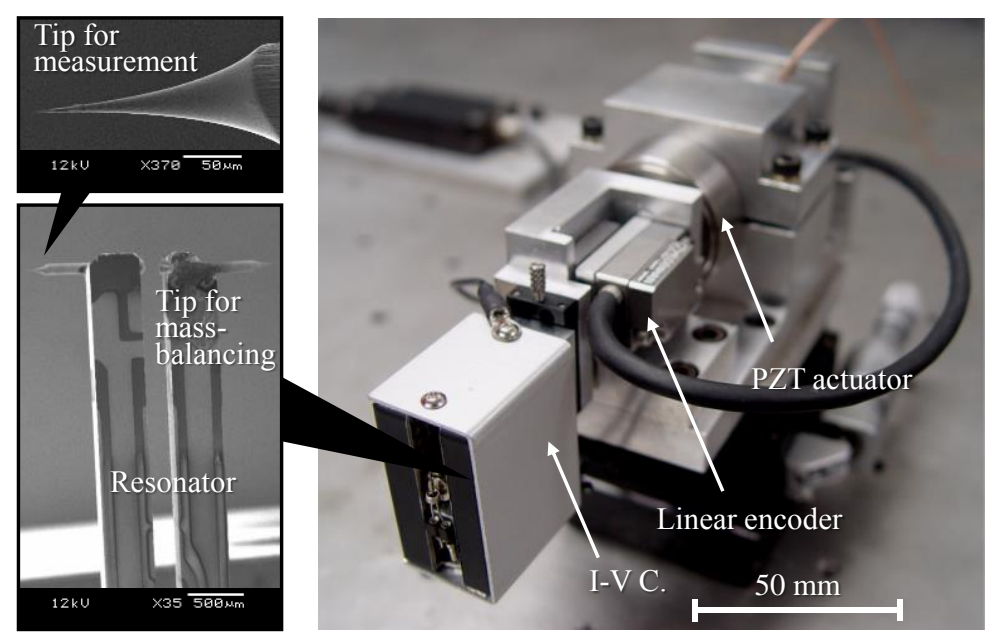

Fig. 4 A photograph of the Z-scanner and SEM images of the probe unit.

\section{Experiments and discussions 4.1 Approach curves}

In order to investigate the feasibilities of the SEFM and the dual-height method, approach curves (distance-frequency shift characteristic curves) were measured. The experiments were conducted in air atmosphere. The temperature and the relative humidity were approximately $300 \mathrm{~K}$ (80 degrees F) and 45\%, respectively. The tip was brought close to the sample at the speed of $100 \mathrm{~nm} / \mathrm{s}$ until the tip contacted the sample surface. The frequency shift was recorded with respect to the displacement of the $\mathrm{Z}$ scanner. The half amplitude of the tip oscillation was set to approximately $10 \mathrm{~nm}$.

Figures 5 and 6 show the obtained approach curves. The lateral axes are relative displacements of the $Z$ actuator. The horizontal axes are frequency shifts which represent $\mathrm{Z}$ directional derivatives of electrostatic forces.

Figures 5 show the approach curves on the glass sample. When the voltage of the electrode was set to $0 \mathrm{~V}$, as can be seen in Fig. 5(a), the approach curve was nearly flat until the tip contacted on the sample. On the other hand, when the voltage of the electrode was set to $50 \mathrm{~V}$, as can be seen in Fig. 5(b), long-range attractive force was observed. The attractive force is well approximated by an inverse-square curve. The estimated tip-sample distance, which was obtained in the least square fitting process of the inverse-square curve, is indicated in Fig. 5(b). According to the estimated tip-sample distance, the tip contacted the samples at the estimated tip-sample distance of around $20 \mathrm{~nm}$, which are close to the half amplitude of the probe oscillation. Therefore, the frequency shift signals on the glass sample were surely confirmed to be inversely proportional to the square of the tip-sample distances. The force curves were measured several times. And the force curves showed the same behavior as Fig. 5(b), indicating that the electrostatic force was stable unless the bias voltage was turned off. The SEFM with the dual-height method is, therefore, applicable to the glass sample. 


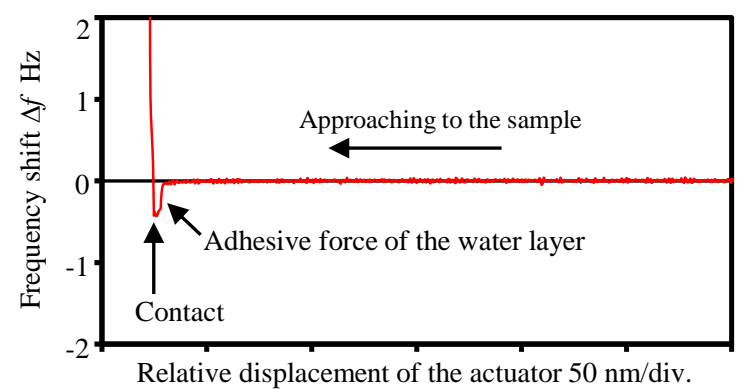

(a) When the voltage of sample electrode was set to $0 \mathrm{~V}$

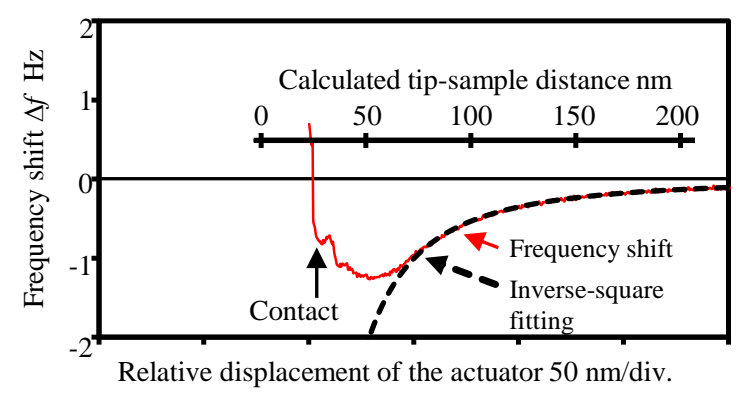

(b) When the voltage of sample electrode was set to $50 \mathrm{~V}$

Fig. 5 Approach curves on the glass sample.

Figures 6 show the approach curves on the acrylic. The approach curves were nearly flat regardless of the voltage of the sample electrode. The tip-sample distance couldn't be estimated because the electrostatic interaction was not observed. The SEFM with the dual-height method is, therefore, not applicable to the acrylic sample. The controllability of the electrostatic force is considered to depend on the hydrophilicity. Judging from the approach curves when the bias voltage was $0 \mathrm{~V}$, only the glass had adhesive force of the water layer. The water layer on the glass would make the electrostatic characteristic of the glass be similar to that of metal (Hu et al. 1995).

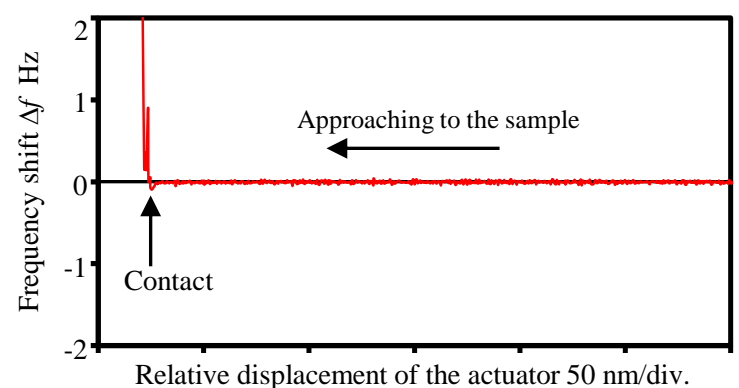

(a) When the voltage of sample electrode was set to $0 \mathrm{~V}$

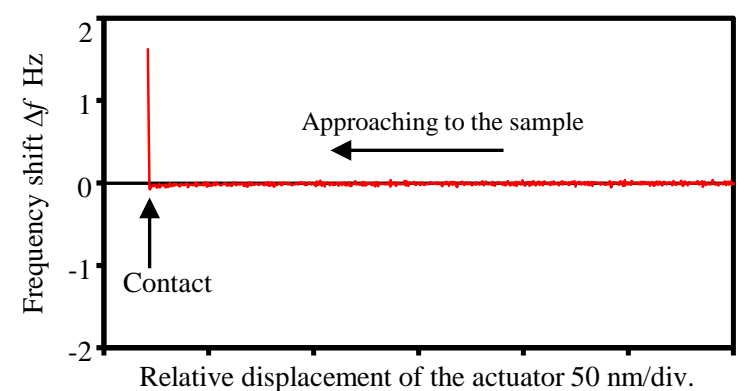

(b) When the voltage of sample electrode was set to $50 \mathrm{~V}$

Fig. 6 Approach curves on the acrylic sample.

\subsection{Surface profile measurement}

Surface topography measurement of the glass sample was conducted. The voltage of the electrode was set to $50 \mathrm{~V}$. The scanning speed was $500 \mathrm{~nm} / \mathrm{s}$. The sampling resolutions in X (scanning direction) and Y axes were $1 \mathrm{~nm}$ and 500 nm respectively. The scanning range was $20 \times 20 \mu \mathrm{m}$. The setpoints of $\Delta f_{a}$ and $\Delta f_{b}$ were set to $-1.7 \mathrm{~Hz}$ and $-1.3 \mathrm{~Hz}$, respectively.

Figures 7 show a series of the measurement data with the dual-height method. Figures 7 (a) and (b) show the tip trajectories of constant interaction scanning when the set point of frequency shift were set to $-1.7 \mathrm{~Hz}$ and $-1.3 \mathrm{~Hz}$, respectively. Figures 7 (c) and (d) show the frequency shift signals obtained with the trajectories $d_{a}$ and $d_{b}$, respectively. The frequency shift images are called 'error maps' because the Z directional position of the tip was feedback controlled to keep the frequency shift constant. The feedback error directly induces profile measurement error in the conventional SPMs. It should be noted that the developed SEFM eliminates the influences of the feedback error, as well as the force intensity distribution, by the algorithm of the dual-height method. Figure 7 (e) shows the tip-sample distance image which was calculated from the tip trajectories and the frequency shifts using Eqs. (8) and (9). It can be seen that the tip-sample distance was maintained larger than $100 \mathrm{~nm}$ during the measurement, realizing completely noncontact scan. Figure 7 (f) shows the surface profile image obtained with Eq. (10). The tip trajectories and the profile image are similar to each other qualitatively. However, quantitative values in $\mathrm{Z}$ direction such as the depth of the valley is different. 


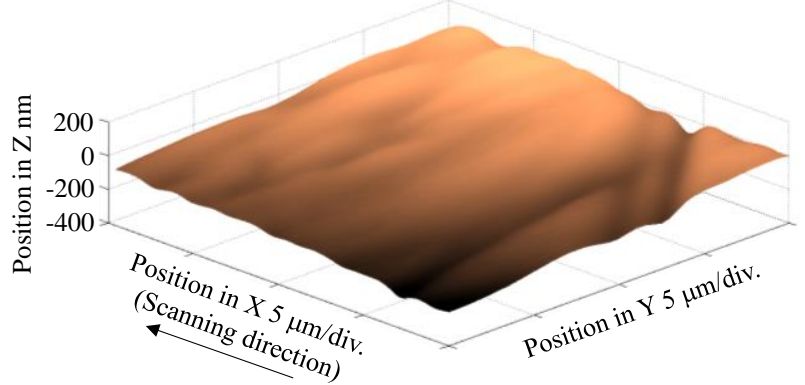

(a) Tip trajectory $d_{a}$ when the setpoint was $-1.7 \mathrm{~Hz}$

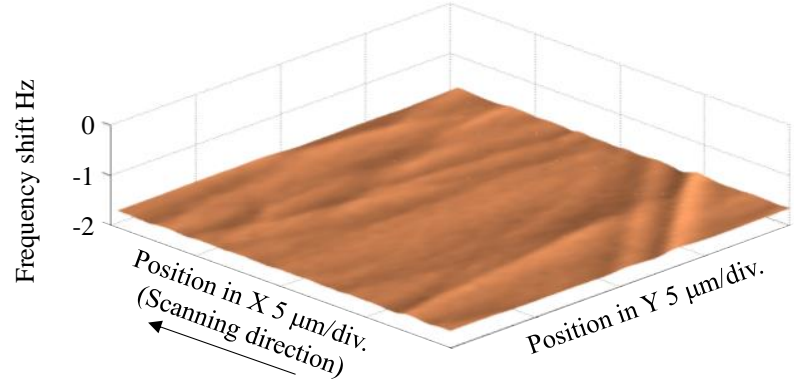

(c) Frequency shift $\Delta f_{a}$ when the setpoint was $-1.7 \mathrm{~Hz}$

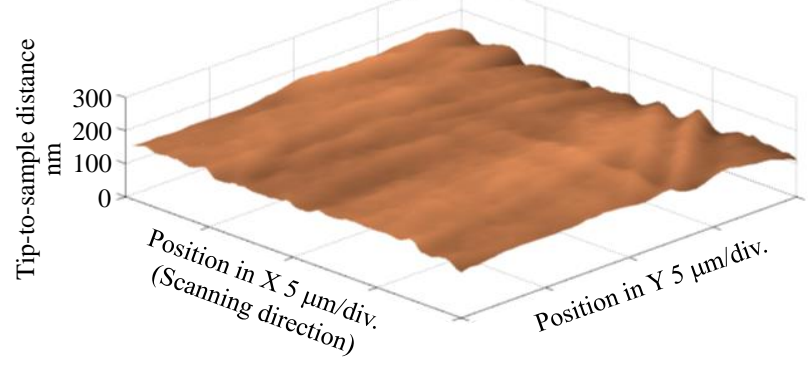

(e) Calculated tip-sample distance

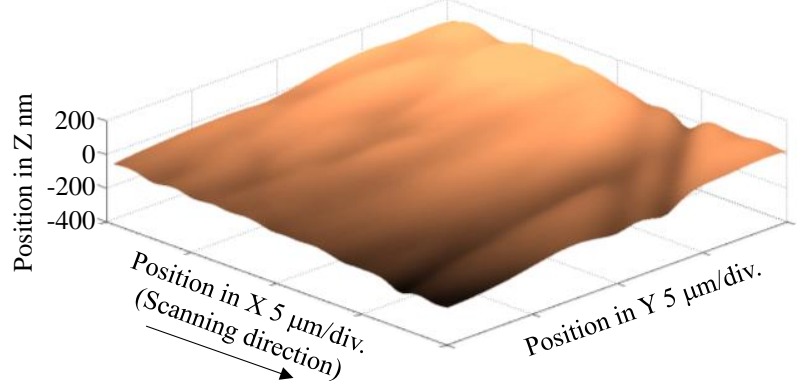

(b) Tip trajectory $d_{b}$ when the setpoint was $-1.3 \mathrm{~Hz}$

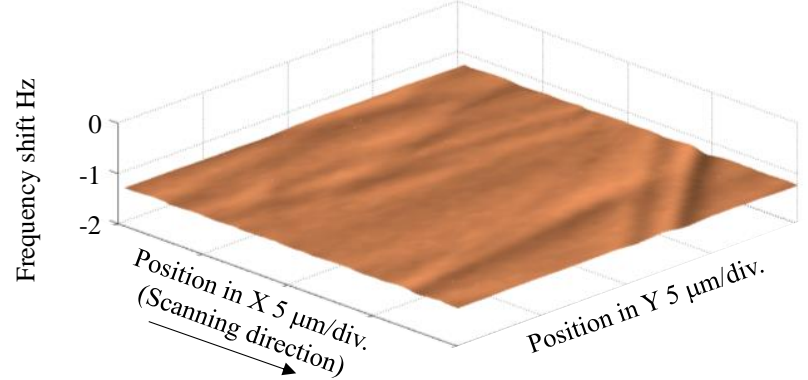

(d) Frequency shift $\Delta f_{a}$ when the setpoint was $-1.3 \mathrm{~Hz}$

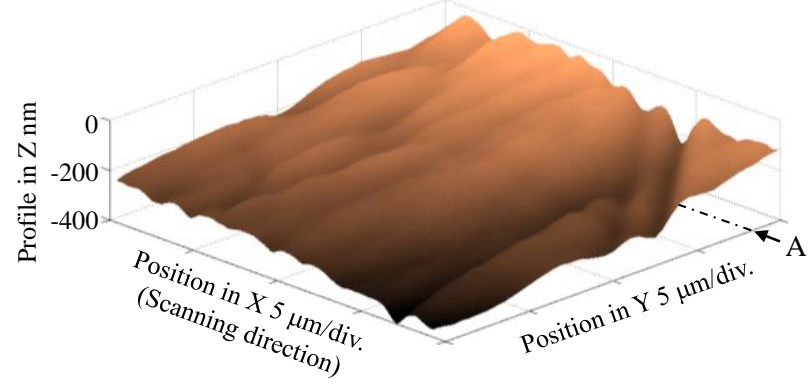

(f) Surface profile image

Fig. 7 Experimental result of noncontact surface profile measurement of the glass. From the images of tip trajectories and the frequency shifts, the tip-sample distance image was created with the dial-height method. Then the surface profile image was created by subtracting the tip-sample distance image from the tip trajectory $d_{a}$.

The developed SEFM can switch the mode to tapping (intermittent contact) AFM mode only by changing the voltage of the electrode to $0 \mathrm{~V}$. Figure 8 shows a tapping AFM image of the same glass sample as Fig. 7 . Figure 9 shows the comparison of the cross-sectional profile images along the scanning directions picked from Figs. 7 and 8. The SEFM tip trajectory did not agree with the AFM profile image quantitatively. However, the SEFM profile image calculated with dual-height method agreed with the AFM profile image quantitatively, showing the effectiveness of the dual-height method on the profile measurement of glass sample.

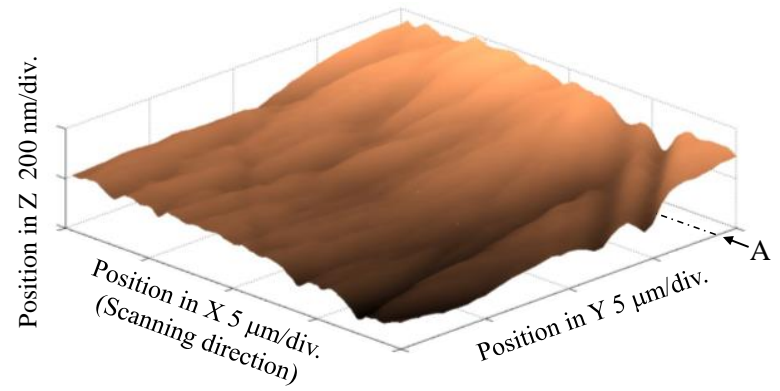

Fig. 8 Tapping AFM topography of the glass 


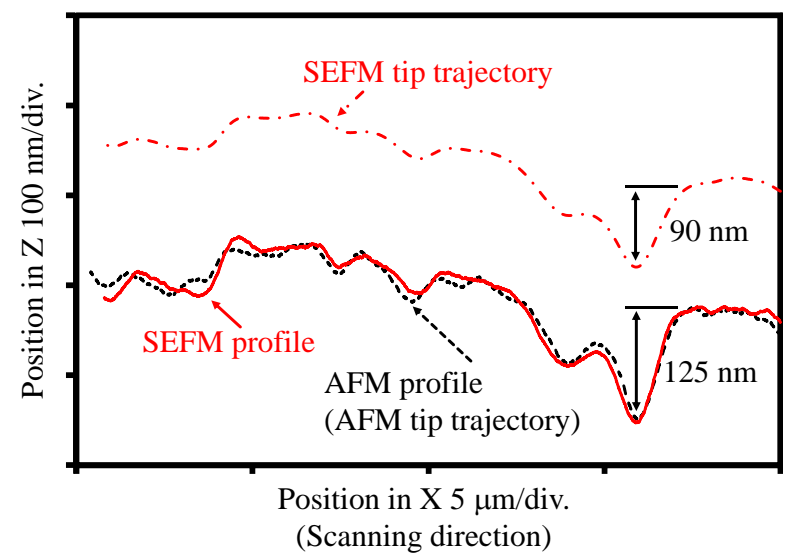

Fig. 9 Comparison of the profile images obtained with the SEFM and the AFM. The scanlines marked with 'A' in Fig. 7 (f) and Fig. 8 are compared.

\section{Conclusions}

A noncontact scanning electrostatic force microscope (SEFM) for surface profile measurement was introduced, and applied to the profile measurement of insulating samples. The SEFM calculates the tip-sample distance with the algorithm named 'dual-height method', and compensates for the fluctuation of the tip-sample distance to obtain accurate surface profile image. Glass and acrylic, which are commonly applied to optical components, were employed as the insulating samples. In the setup for the experiments, the bias voltage was applied to the sample with the electrode clamp in the same manner as the conventional STM system. On the glass sample, electrostatic force was observed in the case that the bias voltage of $50 \mathrm{~V}$ was on. Meanwhile on the acrylic sample, electrostatic force was not observed regardless of the bias voltage.

Surface profile measurement of the glass sample was demonstrated. The tip-sample distance image was created with the dual-height method from the data of the tip trajectory and the frequency shift signal obtained through the constant tip-sample interaction scan. The results showed that the tip-sample distance was kept larger than $100 \mathrm{~nm}$, which is more than ten times larger than that of conventional SPMs. The large tip-sample distance brings the SEFM a high robustness against the disturbances from environment, which would cause tip-sample collision in conventional noncontact SPMs. The same area on the same sample was measured also in the tapping AFM mode, which is realized by setting the bias voltage $0 \mathrm{~V}$. The SEFM image agreed with the AFM image quantitatively, showing that the SEFM with the dual-height method is applicable to noncontact profile measurement of the glass On the other hand, it is difficult to utilize the SEFM in the profile measurement of the acrylic sample because the electrostatic force on the acrylic was not controllable with the voltage of the electrode clamp.

\section{Acknowledgment}

The research was supported by the Japan Society for the Promotion of Science (JSPS).

\section{References}

Barth C., Foster AS., Henry CR. and Shluger AL., Recent Trends in Surface Characterization and Chemistry with HighResolution Scanning Force Methods, Advanced Materials, Vol.23 (2011), pp.477-501.

Belaidi, S., Girard, P. and Leveque, G., Electrostatic Forces Acting on the Tip in Atomic Force Microscopy: Modelization and Comparison with Analytic Expressions, Journal of Applied Physics, Vol.81 (1997), pp.1023-1030.

Binnig G., Rohrer H., Gerber C. and Weibel E., Tunneling through a controllable vacuum gap, Applied Physics Letters, Vol.40, No.2 (1982), pp.178-180.

Binnig G., Quate CF. and Gerber C., Atomic Force Microscope, Physical Review Letters, Vol.56, No.9 (1986), pp.930933.

Gao W., Goto S., Hosobuchi K., Ito S. and Shimizu Y., A noncontact scanning electrostatic force microscope for surface 
profile measurement, CIRP Annals - Manufacturing Technology, Vol.61 (2012), pp.471-474.

Giessibl FJ., Advances in atomic force microscopy, Reviews of Modern Physics, Vol.75 (2003), pp.949-983.

Goto S., Hosobuchi K. and Gao W., An Ultra-Precision Scanning Tunneling Microscope Z-scanner for Surface Profile Measurement of Large Amplitude Micro-Structures, Measurement Science and Technology, Vol.22, No.8 (2011), 085101.

He G., Jia Z., Ito S., Shimizu Y. and Gao W., Experiment of Polarization Forces in Scanning Electrostatic Force Microscopy for Measuring Surface Profile of Dielectric, The Open Electrical \& Electronic Engineering Journal, Vol.8 (2014), pp.342-347.

Hu J., Xiao XD., Salmeron M., Scanning polarization force microscopy: A technique for imaging liquids and weakly adsorbed layers, Applied Physics Letters, Vol.67, No.4 (1995), pp.476-478.

Ng BP., Zhang Y., Kok SW. and Soh YC., Improve performance of scanning probe microscopy by balancing tuning fork prongs, Ultramicroscopy Vol.109 (2009) pp.291-295. 